\title{
Autorenverzeichnis 1992
}

\section{Infusionstherapie 1992; 19:336}

Åkerblom,O. 110,283 Andres, J. 199 Appel, R. suppl 1 Arseniev, L. 199 Asmussen, S. 208

Bacher, C. 276 Ballas, M. 127 Blattmer,K. 199 Baur,A. 73 Becker, H. suppl 1 Becker, K.P. suppl 1 Belia,M. 294 Benzer,A. 181 Blombäck,M. 110,283 Bodenstein, H. suppl 1 Boehm, B.O. 134 Boekstegers, P. suppl 1 Böhme,M. 204 Böker,Ch. 190 Borberg, H. 237, 245, 253 Boughton, B.J. 197 Bouronikou, H. 294 Brauer, P. 232 Bremme,K. 110,283 Burdach, S. suppl 1 Burmester, G.R. suppl 1 Burnouf,T. 91 Burnouf-Radosevich, M. 91

Colombo, T. 171 Cronemeyer, L. 64

Dackland, Å.-L. 283 Darda, C. 12 Dederichs, G. 64 Deller,A. 121 Dengler, T. 76 Denis, R. 291

Dervenoulas, J. 294 Dietrich, G. 151,226 Dietrich, K. 151 Ditschuneit, H.H. 194 Doehn, M. suppl 1 Dongen, W.J.J. van 38

Eckardt,N. 19

Eckart,J. 169

Eckle,R. 151

Eckstein, R. 56,146,149,288

Economopoulos, T. 294

Eiermann, T.H. 127

Emmler, J. 56

Ernst, M. 9,12

Fakler,J. 127 Fatah, K. 283

Fateh-Moghadam, A. suppl 1 Federlin, K. suppl 1 Fellmer, F. 56 Fischer, K. 208 Flegel,W.A. 12,202 Forstner, K. 121 Frank, R. 134 Franz, H.E. 194 Fridrich,L. 181 Fuchs, E. 204 Fürst,G. 76 Gerner, P. 171 Gindi,N. 146 Göbel, U. suppl 1 Goldmann, S.F. 127 Gottardis, M. 181 Graf, A. 130 Grundmann, A. 208 Grunewald, J.M. 194 Grunewald, R.W. 194 Grunewald, S. suppl 1 Gunsilius, E. suppl 1 Gutensohn, K. 19

Hackl,J.M. 181 Haidmann, L. 115 Hänsch,G. 115 Harbauer, G. 23 Härtel,B. 141 Hartert, M. suppl 1 Heim,M. 242 Helmbold,W. 12 Henrich,H.A. 276 Herold,M. 181 Heuckeroth, A. 226 Heuft,H.G. 56 Hinderer, W. 73 Hinghofer-Szalkay, H. 171 Hofman, M.R. 187 Holz, N. suppl 1 Holzberger, G. 134, 157 Horn,J. 73 Horneff, G. suppl 1 Hossfeld, D.-K. 19 Huart,JJ. 91 Jahn,G. 73 Jahn, M. suppl 1 Jungl,E.M. 138

Kääb, S. suppl 1

Kadar,J.G. 237,245

Kalden, J.R. suppl 1

Kapsnen, T. suppl 1

Karakassis, D. 294

Karen, H. 204

Karger, S. 170

Kasulke, D. 12

Kautz,H. 59 
Kellner, S. 76

Kirgis, A. 56

Klein, J. 194

Knödler,B. 19

Knuever-Hopf, J. 79

Koenigsrainer, A. 181

Köhler,T. 187,190

Roller, J. 181

König,W. 194

Konrad,F. 121

Rörholz, D. suppl 1

Rotitschke, R. 23

Rrause,K-P. 249

Kretschmer, V. 53, 64,141, 151,

169,226 Rreutzig, B. 258 Rreuzer, E. suppl 1 Rröll,W. 171 Rron,W. 276

Krüger,W. 19 Kühnl,P. 12, 19

Lambrecht, B. 79 Langer, R. 276 Leititis, J.U. suppl 1 Lenhard,V. 9 Link,H. 199 List,W.F. 171

Mader,J. 115 Manger, B. suppl 1 Marschall,R. 141 Matthes,G. 29,249 Meinert,H. 115 Mempel, W. 242, 268 Meßmer,R. 169 Mingers, A.-M. 105 Mitschulat, H. 70,291 Mohr,H. 79 Moog, R.

61 Moss, R.B. suppl 1 Mueller-Eckhardt, C. 12 Müller, C. 127 Müller,N. 59,70,291 Müller-

Berghaus, G. 187,190

Nebel-Schickel, H. 73 Neudorf, U. suppl 1 Neumann, H.J. 141 Neumeyer, H. 84 Northoff,H. 12, 115,202

Papageorgiou, E.. 294 Pappa,V. 294 Parusel, M. 237, 245 Pawlow, I. 29,249 Peschke, B. 157

Peter, K. 169 Pilz, G. suppl 1 Pirner, K. suppl 1 Poliwoda,H. 199 Poschmann, A. 12,208

Ramschak, H. 171 Raptis, S. 294 Rau,G. 269,276 Reuter, P. 115 Richter,E. 249 Richter, G. 32

Riess,H. 146 Riewald, M. 146 Rubbert, A. suppl 1 Rübo, J. suppl 1

Sachs, V. 104 Scheuermann, E.H. 157 Schleuning, M. 242 Schoeppe,W. 157 Schönitzer, D. 12

Schulzki,T. 141,226 Schunter,F. 12 Schüpphaus, S. 187 Schuster, A. suppl 1 Schütt, K.-H. 12

Schwab, J. suppl 1 Schwartz, D.W.M. 138

Schwarz, S. 32 Schwella,N. 149 Schwenecke, S. 204 Seidl,S. 134, 157 Seifried, E. suppl 1

Serke, S. 288 Shearer, W.T. suppl 1 Sibrowski, W. 232 Siegert,W. 288 Simpson, A.W. 197

Smith, N.A. 197 Sonneborn, H.-H. 3,9, 12,73 Soudavar, F. 253 Spilker, G. suppl 1 Sputtek,A.

269,276 Stahlhut,K 288 Stangel,W. 169,199 Stavrou,A. 294 Steinmetz, A. 32 Stenz, R. 121

Strylogiannis, S. 294 Stuttmann, R. suppl 1 Suontaka, A.-M. 110,283 Suschke, J. suppl 1

Sybesma, J.P.H.B. 38

Taborski,U. 187, 190

Urbaschek, R. suppl 1 Usadel,K-H. 169 Utz,H. 242

Voit, T. suppl 1 Vornhagen, R. 73

Wahn, V. suppl 1 Wank,R 130 Watts, M. 197 Webber, F.E. 138 Weber-Muscan, M.-T. 9

Wehmeier, A. suppl 1 Weidenhöfer, S. suppl 1 Weinstock, C. 202 Weisbach,V. 56,146,288

Weißhaar,D. 258 Werdan, R. suppl 1 Wick, M. suppl 1 Wiebecke, D. 204 Wieding,J.U. 84

Wienhausen, V. 59 Wieser,Ch. 181 Wimmer, A. 130 Wolf, H. 73 Wölpl,A. 127 Wortmann, W. 70

Yurttas, R. 202 
Zander, R. 221 Zeiler,T. 56, 146,288 Zeller,W. 19 Ziemer, S. 29 Zimmermann, R. 56, 288 Zingsem,J. 56, 146, 149,288

336 